\title{
The boundedness of Marcinkiewicz integrals commutators on non-homogeneous metric measure spaces
}

Cao Yonghui and Zhou Jiang*

\author{
*Correspondence: \\ zhoujiangshuxue@126.com \\ College of Mathematics and System \\ Science, Xinjiang University, Urumqi, \\ 830046, P.R. China
}

\begin{abstract}
Let $(\mathcal{X}, d, \mu)$ be a metric measure space satisfying the upper doubling condition and geometrically doubling condition in the sense of Hytönen. In this paper, the authors establish the boundedness of the commutator generated by the $\mathrm{RBMO}(\mu)$ function and the Marcinkiewicz integral with kernel satisfying a Hörmander-type condition, respectively, from $L^{p}(\mu)$ with $1<p<\infty$ to itself.
\end{abstract}

MSC: 42B20; 42B35; 47B47; 30L99

Keywords: non-homogeneous metric measure spaces; Marcinkiewicz integral; commutator; Lebesgue space; $\mathrm{RBMO}(\mu)$

\section{Introduction}

In 1938, Marcinkiewicz [1] introduced the integral on one-dimensional Euclidean space $\mathbb{R}$, which is today called the Marcinkiewicz integral, and conjectured that it is bounded on $L^{p}([0,2 \pi]), 1<p<\infty$. Zygmund in [2] proved the Marcinkiewicz conjecture. In 1958, Stein [3] generalized the above Marcinkiewicz integral to the higher-dimensional case. Let $\Omega$ be homogeneous of degree zero in $\mathbb{R}^{n}, n \geq 2$, integrable and have mean value zero on the unit sphere $\mathcal{S}^{n-1}$. The higher-dimensional Marcinkiewicz integral is then defined by

$$
\mathcal{M}_{\Omega}(f)(x)=\left\{\int_{0}^{\infty}\left|\int_{|x-y| \leq t} \frac{\Omega(x-y)}{|x-y|^{n-1}} f(y) d y\right|^{2} \frac{d t}{t^{3}}\right\}^{\frac{1}{2}}, \quad x \in \mathbb{R}^{n} .
$$

Stein [3] proved that if $\Omega \in \operatorname{Lip}_{\alpha}\left(\mathcal{S}^{n-1}\right)$ for some $\alpha \in(0,1]$, then $\mathcal{M}_{\Omega}$ is bounded on $L^{p}\left(\mathbb{R}^{n}\right)$ for $p \in(1,2]$ and also bounded from $L^{1}\left(\mathbb{R}^{n}\right)$ to $L^{1, \infty}\left(\mathbb{R}^{n}\right)$. Since then, many papers focused on the boundedness of this operator on various function spaces. We refer the reader to [4-12] for its developments and applications.

The main purpose of this paper is to establish the bound of the commutator generated by the Marcinkiewicz integral and the $\operatorname{RBMO}(\mu)$ function on the non-homogeneous metric measure spaces.

During the past 10 to 15 years, considerable attention has been paid to the study of the classical theory of harmonic analysis on Euclidean spaces with non-doubling measures only satisfying the polynomial growth condition (see [13-21]). To be precise, let $\mu$ be a

(c) 2015 Yonghui and Jiang. This article is distributed under the terms of the Creative Commons Attribution 4.0 International License (http://creativecommons.org/licenses/by/4.0/), which permits unrestricted use, distribution, and reproduction in any medium, provided you give appropriate credit to the original author(s) and the source, provide a link to the Creative Commons license, and indicate if changes were made. 
positive Radon measure on $\mathbb{R}^{d}$ with satisfies the polynomial growth condition that, for all $x \in \mathbb{R}^{d}$ and $r>0$,

$$
\mu(B(x, r)) \leq c_{0} r^{n}
$$

where $c_{0}$ is a positive constants and $0<n \leq d$, and $B(x, r)$ is the open ball centered at $x$ and having radius $r$. The analysis associated with such non-doubling measure $\mu$ has proved to play a striking role in solving the long-standing open Painlevés problem and Vitushkin's conjecture by Tolsa [19]. The non-doubling measure $\mu$ may not satisfy the well-known doubling condition, which is a key assumption in harmonic analysis on spaces of homogeneous type in the sense of Coifman and Weiss [22, 23]. To unify both spaces of homogeneous type and the metric spaces endowed with measures satisfying the polynomial growth condition, Hytönen [24] introduced a new class of metric measure spaces satisfying both the so-called geometrically doubling and the upper doubling condition, which are called non-homogeneous metric measure spaces (see Definition 1.3 below). Many classical results have been proved still valid if the underlying spaces are non-homogeneous metric measure spaces (see [25-32]). From now on, we always assume that $(\mathcal{X}, d, \mu)$ is a non-homogeneous metric measure spaces in the sense of Hytönen [24]. In this setting, Hytönen [24] introduced the space $\operatorname{RBMO}(\mu)$, and Hytönen and Martikainen [27] established a version of the Tb theorem. About Marcinkiewicz integral, Lin and Yang [31] have proved that the $L^{p}(\mu)$-boundedness with $p \in(1, \infty)$ is equivalent to either of its boundedness from $L^{1}(\mu)$ into $L^{1, \infty}(\mu)$ or from the atomic Hardy space $H^{1}(\mu)$ (see [28]) to $L^{1}(\mu)$. They also showed that if the Marcinkiewicz integral is bounded from $H^{1}(\mu)$ to $L^{1}(\mu)$, then it is bounded from $L^{\infty}(\mu)$ to $\operatorname{RBLO}(\mu)$ (see [33]), which is a proper subset of $\operatorname{RBMO}(\mu)$. These results essentially improve the existing results in [34].

Now we recall some necessary notions and notation.

The following notion of the geometrically doubling is well known in analysis on metric spaces, which was originally introduced by Coifman and Weiss in $[22,23]$ and is also known as metrically doubling.

Definition 1.1 A metric space $(\mathcal{X}, d)$ is said to be geometrically doubling if there exists some $N_{0} \in \mathbb{N}$ such that, for all balls $B(x, r) \subset \mathcal{X}$, there exists a finite ball covering $\left\{B\left(x_{i}, \frac{r}{2}\right)\right\}_{i}$ of $B(x, r)$ such that the cardinality of this covering is at most $N_{0}$.

Remark 1.2 Let $(\mathcal{X}, d)$ be a metric space. In [24], Hytönen showed that the following statements are mutually equivalent:

(1) $(\mathcal{X}, d)$ is geometrically doubling.

(2) For any $\varepsilon \in(0,1)$ and any ball $B(x, r) \subset \mathcal{X}$, there exists a finite ball covering $\left\{B\left(x_{i}, \varepsilon r\right)\right\}_{i}$ of $B(x, r)$ such that the cardinality of this covering is at most $N_{0} \varepsilon^{-n}$, where $n=\log _{2} N_{0}$.

(3) For any $\varepsilon \in(0,1)$ and any ball $B(x, r) \subset \mathcal{X}$ contains at most $N_{0} \varepsilon^{-n}$ centers of disjoint balls $\left\{B\left(x_{i}, \varepsilon r\right)\right\}_{i}$.

(4) There exists $M \in \mathbb{N}$ such that any ball $B(x, r) \subset \mathcal{X}$ contains at most $M$ centers $\left\{x_{i}\right\}_{i}$ of disjoint balls $\left\{B\left(x_{i}, r / 4\right)\right\}_{i=1}^{M}$.

Definition 1.3 A metric measure space $(\mathcal{X}, d, \mu)$ is said to be upper doubling if $\mu$ is a Borel measure on $\mathcal{X}$ and there exist a dominating function $\lambda: \mathcal{X} \times(0, \infty) \rightarrow(0, \infty)$ and a 
positive constant $c_{\lambda}$ such that, for each $x \in \mathcal{X}, r \rightarrow \lambda(x, r)$ is non-decreasing and

$$
\mu(B(x, r)) \leq \lambda(x, r) \leq c_{\lambda} \lambda(x, r / 2), \quad \text { for all } x \in \mathcal{X}, r>0 .
$$

It was proved in [28] that there exists a dominating function $\tilde{\lambda}$ related to $\lambda$ satisfying the property that there exists a positive constant $c_{\tilde{\lambda}}$ such that $\tilde{\lambda} \leq \lambda, c_{\tilde{\lambda}} \leq c_{\lambda}$ and, for all $x, y \in \mathcal{X}, r>0$ with $d(x, y) \leq r, \tilde{\lambda}(x, r) \leq c_{\tilde{\lambda}} \tilde{\lambda}(y, r)$. Based on this, in this paper, we always assume that the dominating function $\lambda$ also satisfies it.

The following coefficients $\delta(B, S)$ for all ball $B$ and $S$ were introduced in [24] as analogs of Tolsa's number $K_{B, S}$ in [18].

Definition 1.4 For all balls $B \subset S$, let

$$
\delta(B, S)=1+\int_{(2 S-B)} \frac{d \mu(x)}{\lambda\left(c_{B}, d\left(x, c_{B}\right)\right)},
$$

where above and in that follows, for a ball $B=B\left(c_{B}, r_{B}\right)$ and $\rho>0, \rho B=B\left(c_{B}, \rho r_{B}\right)$.

Remark 1.5 The following discrete version $K_{B, S}$ of $\delta(B, S)$ was first introduced by Bui and Duong [25] in non-homogeneous metric measure spaces, which is more close to the quantity $K_{B, S}$ introduced by Tolsa [18] in the setting of non-doubling measures. For all balls $B \subset S$, let $K_{B, S}$ be defined by

$$
K_{B, S}=1+\sum_{i=1}^{N_{B, S}} \frac{\mu\left(6^{i} B\right)}{\lambda\left(c_{B}, 6^{i} r_{B}\right)},
$$

where $N_{B, S}$ denote the smallest integer satisfying $6^{N_{B, S}} r_{B} \geq r_{s}$. Obviously $\delta(B, S) \lesssim K_{B, S}$. As was pointed out by Bui and Duong [25], it is not true that $\delta(B, S) \sim K_{B, S}$.

Definition 1.6 Let $\alpha, \beta \in(0, \infty)$. A ball $B \subset \mathcal{X}$ is called $(\alpha, \beta)$-doubling if $\mu(\alpha B) \leq \beta \mu(B)$.

It was proved in [24] that if a metric measure space $(\mathcal{X}, d, \mu)$ is upper doubling and $\alpha, \beta \in(0, \infty)$ satisfying $\beta>c_{\lambda}^{\log _{2} \alpha}=\alpha^{v}$, then, for any ball $B$, there exists some $j \in \mathbb{N} \cup\{0\}$ such that $\alpha^{j} B$ is $(\alpha, \beta)$-doubling. Moreover, let $(\mathcal{X}, d, \mu)$ be geometrically doubling, $\beta>\alpha^{n}$ with $n=\log _{2} N_{0}$ and $\mu$ a Borel measure on $\mathcal{X}$ which is finite on bounded sets. Hytönen [24] also showed that for $\mu$-almost every $x \in \mathcal{X}$, there exist arbitrary small $(\alpha, \beta)$-doubling balls centered at $x$. Furthermore, the radii of these balls may be chosen to be of the form $\alpha^{-j} B$ for $j \in \mathbb{N}$ and any preassigned number $r>0$. Throughout this paper, for any $\alpha \in(1, \infty)$ and ball $B$, the smallest $\left(\alpha, \beta_{\alpha}\right)$-doubling ball of the form $\alpha^{j} B$ with $j \in \mathbb{N}$ is denoted by $\tilde{B}^{\alpha}$, where

$$
\beta_{\alpha}=\max \left\{\alpha^{3 n}, \alpha^{3 v}\right\}+30^{n}+30^{v}
$$

In what follows, by a doubling ball we mean a $\left(6, \beta_{6}\right)$-doubling ball and $\tilde{B}^{6}$ is simply denoted by $\tilde{B}$.

Now we recall the definition of $\operatorname{RBMO}(\mu)$ from [24]. 
Definition 1.7 Let $\rho \in(1, \infty)$. A function $f \in L_{\text {loc }}^{1}(\mu)$ is said to be in the space $\operatorname{RBMO}(\mu)$ if there exist a positive constant $c$ and, for any ball $B \subset \mathcal{X}$, a number $f_{B}$ such that

$$
\frac{1}{\mu(\rho B)} \int_{B}\left|f(x)-f_{B}\right| d \mu(x) \leq c
$$

and, for any two balls $B \subset S$,

$$
\left|f_{B}-f_{S}\right| \leq c \delta_{B, S}
$$

The infimum of the positive constant $c$ is defined to be the $\operatorname{RBMO}(\mu)$ norm of $f$ and denote by $\|f\|_{\operatorname{RBM}(\mu)}$.

In [24], it follows that the definition of $\operatorname{RBMO}(\mu)$ is independent of the choice of $\rho \in$ $(1, \infty)$.

The following equivalent characterization of $\operatorname{RBMO}(\mu)$ was established in [28].

Lemma 1.8 Let $\rho \in(1, \infty)$ and $f \in L_{\mathrm{loc}}^{1}(\mu)$. Then the following statements are equivalent:

(1) $f \in \operatorname{RBMO}(\mu)$;

(2) there exist a positive constant $c$ and, for any ball $B \subset \mathcal{X}$, such that

$$
\frac{1}{\mu(\rho B)} \int_{B}\left|f(x)-m_{\tilde{B}} f\right| d \mu(x) \leq c
$$

and, for any doubling balls $B \subset S$,

$$
\left|m_{B}-m_{S}\right| \leq c \delta_{B, S}
$$

Moreover, let $\|f\|_{*}$ be the infimum of the positive constant $c$ in (2). Then there exists a constant $\tilde{c}$ such that $\frac{\|f\|_{*}}{\tilde{c}} \leq\|f\|_{\mathrm{RBMO}(\mu)} \leq \tilde{c}\|f\|_{*}$.

Now we give the definition of Marcinkiewicz integral (see [31]).

Definition 1.9 Let $K$ be a locally integrable function on $(\mathcal{X} \times \mathcal{X}) \backslash\{(x, x): x \in \mathcal{X}\}$. Assume that there exists a positive constant $c$ such that, for all $x, y \in \mathcal{X}$ with $x \neq y$,

$$
|K(x, y)| \leq c \frac{d(x, y)}{\lambda(x, d(x, y))}
$$

and, for all $y, y^{\prime} \in \mathcal{X}$,

$$
\int_{d(x, y) \geq 2 d\left(y, y^{\prime}\right)}\left[\left|K(x, y)-K\left(x, y^{\prime}\right)\right|+\left|K(y, x)-K\left(y^{\prime}, x\right)\right|\right] \frac{1}{d(x, y)} d \mu(x) \leq c .
$$

The Marcinkiewicz integral $\mathcal{M} f$ associated to the above kernel $K$ is defined by setting, for all $x \in \mathcal{X}$,

$$
\mathcal{M}(f)(x)=\left[\int_{0}^{\infty}\left|\int_{d(x, y)<t} K(x, y) f(y) d \mu(y)\right|^{2} \frac{d t}{t^{3}}\right]^{\frac{1}{2}} .
$$


We show that the commutator $\mathcal{M}_{b}$, associating with $b \in \operatorname{RBMO}(\mu)$ and $\mathcal{M}$, which is defined by

$$
\mathcal{M}_{b}(f)(x)=[b, \mathcal{M}](f)(x)=b(x) \mathcal{M}(f)(x)-\mathcal{M}(b f)(x), \quad x \in \mathcal{X}
$$

In [31], the authors have proven that the Marcinkiewicz integral $\mathcal{M}$ is bounded from $L^{p}(\mu)$ to $L^{p}(\mu), 1<p<\infty$. Our main result is as follows.

Theorem 1.10 Let $K$ satisfy (1.5) and the following Hörmander-type condition:

$$
\begin{aligned}
& \sup _{r>0, d\left(y, y^{\prime}\right) \leq r} \sum_{i=1}^{\infty} i \int_{6^{i} r<d(x, y) \leq 6^{i+1} r}\left[\left|K(x, y)-K\left(x, y^{\prime}\right)\right|\right. \\
& \left.+\left|K(y, x)-K\left(y^{\prime}, x\right)\right|\right] \frac{1}{d(x, y)} d \mu(x) \leq c .
\end{aligned}
$$

If $\mathcal{M}$ is bounded on $L^{2}(\mu)$, then, for any $b \in \operatorname{RBMO}(\mu)$, the commutator $\mathcal{M}_{b}$ is bounded on $L^{p}(\mu)$ with the bound no more than $c_{p}\|b\|_{\mathrm{RBMO}(\mu)}$, where $1<p<\infty$.

Remark 1.11 The Hörmander-type condition (1.9) is slightly stronger than (1.6).

The organization of this paper is as follows. In Section 2, we introduce the sharp maximal operator $M^{\#}$, associated with $K_{B, S}$ and prove Lemma 2.6. This technical lemma is of independent interest. Section 3 is devoted to the proof of Theorem 1.10.

Throughout this paper, we denote $c$ a positive constant which is independent of the main parameters involved, but may vary from line to line. For any ball $B \subset \mathcal{X}$, we denote its center and radius by $c_{B}$ and $r_{B} . m_{B} f$ means that $\frac{1}{\mu(B)} \int_{B} f(y) d \mu(y)$.

\section{The sharp maximal function}

For a locally integrable function $f$, let $M^{\#} f$ be the sharp maximal function of $f$, namely, for $x \in \mathcal{X}$

$$
M^{\#} f(x)=\sup _{x \in B} \frac{1}{\mu(6 B)} \int_{B}\left|f(y)-m_{\tilde{B}} f\right| d \mu(y)+\sup _{(B, S) \in \Delta_{x}} \frac{\left|m_{B} f-m_{S} f\right|}{K_{B, S}},
$$

where $\Delta_{x}=\{(B, S): x \in B \subset S$ and $B, S$ are doubling balls $\}$.

For $0<r<\infty$, let $M_{r}^{\#} f(x)=\left[M^{\#}\left(|f|^{r}\right)(x)\right]^{\frac{1}{r}}$ for $x \in \mathcal{X}$. A simple computation proves that if $0<r<1$,

$$
M_{r}^{\#} f(x) \leq c_{r} M^{\#} f(x)
$$

where $c_{r}>0$ is independent of $f$ and $x$.

We recall some results in [32].

\section{Lemma 2.1}

(1) Let $p \in(1, \infty), r \in(1, p)$ and $\rho \in[5, \infty)$. The following maximal operators defined, respectively, by setting, for all $f \in L_{\mathrm{loc}}^{1}(\mu)$ and $x \in \mathcal{X}$ :

$$
M_{r, \rho} f(x)=\sup _{x \in B}\left\{\frac{1}{\mu(\rho B)} \int_{B}|f(y)|^{r} d \mu(y)\right\}^{\frac{1}{r}},
$$




$$
N f(x)=\sup _{x \in B: \text { doubling }} \frac{1}{\mu(B)} \int_{B}|f(y)| d \mu(y)
$$

and

$$
M_{\rho} f(x)=\sup _{x \in B} \frac{1}{\mu(\rho B)} \int_{B}|f(y)| d \mu(y)
$$

are bounded on $L^{p}(\mu)$ and also bounded from $L^{1}(\mu)$ to $L^{1, \infty}(\mu)$.

(2) For all $f \in L_{\text {loc }}^{1}(\mu)$ it holds true that $|f(x)| \leq N f(x)$ for $\mu$-almost every $x \in \mathcal{X}$.

In Lemma 2.1, if $0<r<1$, using the Hölder inequality, we have $M_{r, \rho} f(x)<M_{\rho} f(x)$. So Lemma 2.1 is right when $0<r<1$.

We also need the following Calderón-Zygmund decomposition theorem obtained by Bui and Duong [25]. Let $\gamma$ be a fixed positive constant satisfying that $\gamma>\max \left\{c_{\lambda}^{3 \log _{2} 6}, 6^{3 n}\right\}$ where $c_{\lambda}$ is as in Definition 1.3 and $n$ as in Remark 1.2.

Lemma 2.2 Let $p \in[1, \infty), f \in L^{p}(\mu)$ and $t \in(0, \infty)\left(t>\frac{\gamma^{\frac{1}{p}}\|f\|_{L^{p}}(\mu)}{[\mu(\mathcal{X})]^{1 / p}}\right.$ when $\left.\mu(\mathcal{X})<\infty\right)$. Then

(1) there exists a family of finite overlapping balls $\left\{B_{j}\right\}_{j}$, pairwise disjoint,

$$
\begin{aligned}
& \frac{1}{\mu\left(6^{2} B_{j}\right)} \int_{B_{j}}|f(x)|^{p} d \mu(x)>\frac{t^{p}}{\gamma} \quad \text { for all } j, \\
& \frac{1}{\mu\left(6^{2} \eta B_{j}\right)} \int_{\eta B_{j}}|f(x)|^{p} d \mu(x) \leq \frac{t^{p}}{\gamma} \quad \text { for all } j \text { and all } \eta \in(2, \infty),
\end{aligned}
$$

and

$$
|f(x)| \leq t \quad \text { for } \mu \text {-almost every } x \in \mathcal{X} \backslash\left(\bigcup_{j} 6 B_{j}\right)
$$

(2) for each $j$, let $S_{j}$ be $a\left(3 \times 6^{2}, c_{\lambda}^{\log _{2} 3 \times 6^{2}+1}\right)$-doubling ball of the family $\left\{\left(3 \times 6^{2}\right)^{k} B_{j}\right\}_{k \in \mathbb{N}}$, and $\omega_{j}=\frac{\chi_{6 B_{j}}}{\sum_{k} \chi_{6 B_{k}}}$. Then there exists a family $\left\{\varphi_{j}\right\}_{j}$ of functions such that, for each $j$, $\operatorname{supp}\left(\varphi_{j}\right) \subset S_{j}, \varphi_{j}$ has a constant sign on $S_{j}$,

$$
\begin{aligned}
& \int_{\mathcal{X}} \varphi_{j}(x) d \mu(x)=\int_{6 B_{j}} f(x) \omega_{j}(x) d \mu(x), \\
& \sum_{j}\left|\varphi_{j}(x)\right| \leq \gamma_{0} t \quad \text { for } \mu \text {-almost every } x \in \mathcal{X},
\end{aligned}
$$

where $\gamma_{0}$ is some positive constant depending only on $(\mathcal{X}, \mu)$, and there exists a positive constant $c$, independent off, $t$ and $j$ such that, when $p=1$, it holds true that

$$
\left\|\varphi_{j}\right\|_{L^{\infty}(\mu)} \mu\left(S_{j}\right) \leq c \int_{\mathcal{X}}\left|f(x) \omega_{j}(x)\right| d \mu(x)
$$

and, if $p \in(1, \infty)$, it holds true that

$$
\left\{\int_{S_{j}}\left|\varphi_{j}(x)\right|^{p} d \mu(x)\right\}^{1 / p}\left[\mu\left(S_{j}\right)\right]^{1 / p^{\prime}} \leq \frac{c}{t^{p-1}} \int_{\mathcal{X}}\left|f(x) \omega_{j}(x)\right|^{p} d \mu(x) .
$$


The following John-Nirenberg inequality was established by Hytönen in [24].

Lemma 2.3 let $(\mathcal{X}, d, \mu)$ be geometrically doubling and upper doubling. For every $\rho>1$, there is a constant $c$ so that, for every $f \in \operatorname{RBMO}(\mu)$ and every ball $B$,

$$
\mu\left(x \in B:\left|f(x)-f_{B}\right|>t\right) \leq 2 \mu(\rho B) \exp \left(-c t /\|f\|_{\mathrm{RBMO}(\mu)}\right),
$$

where $f_{B}$ can be seen in definition of $\operatorname{RBMO}(\mu)$.

From Lemma 2.3, it is easy to prove that there are two positive $c_{1}, c_{2}$ such that, for any ball $\mathrm{B}$ and $b \in \operatorname{RBMO}(\mu)$,

$$
\frac{1}{\mu(\rho B)} \int_{B} \exp \left(\frac{\left|b(x)-m_{\widetilde{B}}(b)\right|}{c_{1}\|b\|_{\mathrm{RBMO}(\mu)}}\right) d \mu(x) \leq c_{2} .
$$

Lemma 2.4 There is a constant $c$ such that, for any $a>0$ and $t_{1}, t_{2}>0$,

$$
t_{1} t_{2} \leq c\left[t_{1} \log \left(2+a t_{1}\right)+a^{-1} \exp t_{2}\right]
$$

This lemma had been established in [34].

We also need some useful properties of $K_{B, S}$, which were proved in [25, 32].

\section{Lemma 2.5}

(1) For all balls $B \subset R \subset S, K_{B, R} \leq 2 K_{B, S}$.

(2) For any $\rho \in[1, \infty)$, there exists a positive constant $c_{\rho}$, depending only on $\rho$, such that, for all balls $B \subset S$ with $r_{S} \leq \rho r_{B}, K_{B, S} \leq c_{\rho}$.

(3) There exists a positive constant $c$, such that, for all balls $B, K_{B, \tilde{B}} \leq c$.

(4) There exists a positive constant $c$, depending on $c_{\lambda}$, such that, for all balls $B \subset R \subset S$, $K_{B, S} \leq K_{B, R}+c K_{R, S}$.

(5) There exists a positive constant c, depending on $c_{\lambda}$, such that, for all balls $B \subset R \subset S$, $K_{R, S} \leq c K_{B, S}$.

Now we give and prove the main result about the sharp maximal function $M^{\#}$.

Lemma 2.6 Let $K$ satisfy (1.5) and the Hörmander-type condition (1.9). We have $s \in(1, \infty)$, $p_{0} \in(1, \infty)$ and $b \in L^{\infty}(\mu)$. If $\mathcal{M}$ is bounded on $L^{2}(\mu)$, then there is a positive constant $c$ such that, for all $f \in L^{\infty}(\mu) \cap L^{p_{0}}(\mu)$ and for all $x \in \mathcal{X}$,

$$
M^{\#}\left[\mathcal{M}_{b}(f)\right](x) \leq c\left[\|b\|_{\mathrm{RBMO}(\mu)} M_{s, 6}[\mathcal{M}(f)](x)+\|b\|_{\mathrm{RBMO}(\mu)}\|f\|_{L^{\infty}(\mu)}\right] .
$$

Proof Without loss of generality, we may assume $\|b\|_{\mathrm{RBMO}(\mu)}=1$. To prove Lemma 2.6, it suffices to prove that

$$
\frac{1}{\mu(6 B)} \int_{B}\left|\mathcal{M}_{b}(f)(y)-h_{B}\right| d \mu(y) \leq c M_{s, 6}[\mathcal{M}(f)](x)+\|f\|_{L^{\infty}(\mu)}
$$

for all $x \in B$ and

$$
\left|h_{B}-h_{S}\right| \leq c\left(K_{B, S}\right)^{2}\left[M_{s, 6}[\mathcal{M}(f)](x)+\|f\|_{L^{\infty}(\mu)}\right]
$$


for all balls $B \subset S$ with $x \in B$, where $B$ is an arbitrary ball and $S$ is a doubling ball,

$$
h_{B}=m_{B}\left[\mathcal{M}\left(\left(b-m_{\widetilde{B}}(b)\right) f \chi_{\mathcal{X} \backslash \frac{6}{5} B}\right)\right]
$$

and

$$
h_{S}=m_{S}\left[\mathcal{M}\left(\left(b-m_{S}(b)\right) f \chi_{\mathcal{X} \backslash \frac{6}{5} S}\right)\right]
$$

To prove (2.4), for a fixed ball $B, x \in B$ and $f \in L^{\infty}(\mu)$, we write

$$
f(y)=f(y) \chi_{\frac{6}{5} B}(y)+f(y) \chi_{\mathcal{X} \backslash \frac{6}{5} B}(y)=f_{1}(y)+f_{2}(y)
$$

and

$$
\mathcal{M}_{b}(f)(y)=\left(b(y)-m_{\tilde{B}}(b)\right) \mathcal{M}(f)(y)-\mathcal{M}\left(\left(b(y)-m_{\tilde{B}}(b)\right) f_{1}\right)(y)-\mathcal{M}\left(\left(b(y)-m_{\tilde{B}}(b)\right) f_{2}\right)(y) .
$$

So we can write

$$
\begin{aligned}
& \frac{1}{\mu(6 B)} \int_{B}\left|\mathcal{M}_{b}(f)(y)-h_{B}\right| d \mu(y) \\
& \leq \frac{1}{\mu(6 B)} \int_{B}\left|b(y)-m_{\tilde{B}}(b)\right| \mathcal{M}(f)(y) d \mu(y) \\
& \quad+\frac{1}{\mu(6 B)} \int_{B} \mathcal{M}\left(\left(b(y)-m_{\tilde{B}}(b)\right) f_{1}\right)(y) d \mu(y) \\
& \quad+\frac{1}{\mu(6 B)} \int_{B}\left|\mathcal{M}\left(\left(b(y)-m_{\tilde{B}}(b)\right) f_{2}\right)(y)-h_{B}\right| d \mu(y) \\
& =A_{1}+A_{2}+A_{3} .
\end{aligned}
$$

By the Hölder inequality and Corollary 2.3 in [32], we see that

$$
\begin{aligned}
A_{1} & \leq \frac{1}{\mu(6 B)^{\frac{1}{s}+\frac{1}{s^{\prime}}}}\left[\int_{B}\left|b(y)-m_{\tilde{B}}(b)\right|^{s^{\prime}} d \mu(y)\right]^{1 / s^{\prime}}\left[\int_{B}(\mathcal{M}(f))^{s}(y)\right]^{1 / s} \\
& \leq M_{s, 6}[\mathcal{M}(f)](x) .
\end{aligned}
$$

To estimate $A_{2}$, from the Hölder inequality, the $L^{2}(\mu)$-boundedness of $\mathcal{M}$ and Corollary 2.3 in [32], it follows that

$$
\begin{aligned}
A_{2} & \leq \frac{\mu(B)^{1 / 2}}{\mu(6 B)}\left[\int_{B}\left|\mathcal{M}\left[\left(b(y)-m_{\tilde{B}}(b)\right) f_{1}\right](y)\right|^{2} d \mu(y)\right]^{1 / 2} \\
& \leq\left[\frac{1}{\mu(6 B)} \int_{B}\left|\left(b(y)-m_{\tilde{B}}(b)\right) f_{1}(y)\right|^{2} d \mu(y)\right]^{1 / 2} \\
& \leq \mu(6 B)^{-1 / 2}\left\|\left(b(y)-m_{\frac{\sigma_{5} B}{5}}(b)\right) f_{1}(y)\right\|_{L^{2}(\mu)}+\mu(6 B)^{-1 / 2}\left\|\left(m_{\widetilde{\frac{\sigma}{5} B}}(b)-m_{\tilde{B}}(b)\right) f_{1}(y)\right\|_{L^{2}(\mu)} \\
& \leq\|f\|_{L^{\infty}(\mu)}\left[\frac{1}{\mu(6 B)} \int_{\frac{6}{5} B}\left|b(y)-m_{\widetilde{\frac{\sigma}{5} B}}(b)\right|^{2} d \mu(y)\right]^{1 / 2}
\end{aligned}
$$




$$
\begin{aligned}
& \quad+c\|f\|_{L^{\infty}(\mu)}\left[\frac{\mu\left(\frac{6}{5} B\right)}{\mu(6 B)}\right]^{1 / 2} \\
& \leq c\|f\|_{L^{\infty}(\mu)},
\end{aligned}
$$

where we use the fact that $\left|m_{\frac{\widetilde{6}}{5} B}(b)-m_{\tilde{B}}(b)\right| \leq c\left(K_{B, \widetilde{B}}+K_{\frac{6}{5} B, \frac{\widetilde{6}}{5} B}+K_{B, \frac{6}{5} B}\right) \leq c$.

To obtain (2.4), we still need to estimate $A_{3}$. Set

$$
\begin{aligned}
& M_{1}(x, y)=\left(\int_{0}^{\infty}\left[\int_{d(y, z) \leq t \leq d(x, z)}\left|K(y, z)\left(b(z)-m_{\widetilde{B}}(b)\right) f_{2}(z)\right| d \mu(z)\right]^{2} \frac{d t}{t^{3}}\right)^{1 / 2}, \\
& M_{2}(x, y)=\left(\int_{0}^{\infty}\left[\int_{d(x, z) \leq t \leq d(y, z)}\left|K(y, z)\left(b(z)-m_{\widetilde{B}}(b)\right) f_{2}(z)\right| d \mu(z)\right]^{2} \frac{d t}{t^{3}}\right)^{1 / 2},
\end{aligned}
$$

and

$$
M_{3}(x, y)=\left(\int_{0}^{\infty}\left[\int_{\max \{d(y, z), d(x, z)\}}\left|(K(y, z)-K(x, z))\left(b(z)-m_{\widetilde{B}}(b)\right) f_{2}(z)\right| d \mu(z)\right]^{2} \frac{d t}{t^{3}}\right)^{1 / 2} .
$$

For any $x, y \in \mathcal{X}$, we have (see also [31], p.134)

$$
\left|\mathcal{M}\left[\left(b-m_{\widetilde{B}}(b)\right) f_{2}\right](y)-\mathcal{M}\left[\left(b-m_{\widetilde{B}}(b)\right) f_{2}\right](x)\right| \leq \sum_{i=1}^{3} M_{i}(x, y)
$$

Applying the Minkowski inequality and (1.9) we conclude that, for all $x, y \in B$,

$$
\begin{aligned}
& M_{1}(x, y) \leq \int_{d(y, z)<d(x, z)}\left|K(y, z) f_{2}(z)\left(b(z)-m_{\widetilde{B}}(b)\right)\right|\left[\int_{d(y, z) \leq t<d(x, z)} \frac{d t}{t^{3}}\right]^{1 / 2} d \mu(z) \\
& \leq c \int_{\mathcal{X} \backslash 5 B} \frac{r_{B}^{1 / 2}}{d\left(z, c_{B}\right)^{1 / 2}} \frac{\left|b(z)-m_{\widetilde{B}}(b)\right|}{\lambda\left(c_{B}, d\left(z, c_{B}\right)\right)} f(z) d \mu(z) \\
& \leq c \sum_{i=1}^{\infty} \int_{6^{i} 5 B \backslash 6^{i-1} 5 B} \frac{r_{B}^{1 / 2}}{d\left(z, c_{B}\right)^{1 / 2}} \frac{\left|m_{6_{\widetilde{5}} B}(b)-m_{\widetilde{B}}(b)\right|}{\lambda\left(c_{B}, d\left(z, c_{B}\right)\right)} f(z) d \mu(z) \\
& +\sum_{i=1}^{\infty} \int_{6^{i} 5 B \backslash 6^{i-1} 5 B} \frac{r_{B}^{1 / 2}}{d\left(z, c_{B}\right)^{1 / 2}} \frac{\left|m_{6^{\widetilde{5}} \widetilde{F}_{B}}(b)-b(z)\right|}{\lambda\left(c_{B}, d\left(z, c_{B}\right)\right)} f(z) d \mu(z) \\
& \leq c \sum_{i=1}^{\infty} i 6^{(-i / 2)} \frac{1}{\lambda\left(c_{B}, 6^{i-1} 5 r_{B}\right)} \int_{6^{i} 5 B}|f(z)| d \mu(z) \\
& +\sum_{i=1}^{\infty} 6^{(-i / 2)} \frac{1}{\lambda\left(c_{B}, 6^{i-1} 5 r_{B}\right)} \int_{6^{i} 5 B}\left|m_{\sigma^{\widetilde{i} 5 B}}(b)-b(z)\right||f(z)| d \mu(z) \\
& \leq c\|f\|_{L^{\infty}(\mu)} \sum_{i=1}^{\infty} i 6^{(-i / 2)} \frac{\mu\left(6^{i+1} 5 B\right)}{\lambda\left(c_{B}, 6^{i-1} 5 r_{B}\right)} \\
& \leq c\|f\|_{L^{\infty}(\mu)} \text {, }
\end{aligned}
$$

where we use the doubling condition of $\lambda,\left|m_{\sigma_{\sigma_{5}}}(b)-m_{\widetilde{B}}(b)\right| \leq c i$ and $\lambda\left(c_{B}, d\left(x, c_{B}\right)\right) \backsim$ $\lambda\left(x, d\left(x, c_{B}\right)\right) \backsim \lambda(x, d(x, y))$ for $y \in B$ and $x \in \mathcal{X} \backslash k B(k>1)$. 
Similarly, $M_{2}(x, y) \leq c\|f\|_{L^{\infty}(\mu)}$. Now for all $x, y \in B$, by the Minkowski inequality we have

$$
\begin{aligned}
& M_{3}(x, y) \\
& \leq \int_{\mathcal{X}}\left|(K(y, z)-K(x, z)) f_{2}(z)\left(b(z)-m_{\widetilde{B}}(b)\right)\right|\left[\int_{\max \{d(y, z), d(x, z)\} \leq t} \frac{d t}{t^{3}}\right]^{1 / 2} d \mu(z) \\
& \leq c \int_{\mathcal{X}}\left|(K(y, z)-K(x, z)) f_{2}(z)\left(b(z)-m_{\widetilde{B}}(b)\right)\right| \frac{1}{d(y, z)} d \mu(z) \\
& \leq c\|f\|_{L^{\infty}(\mu)} \sum_{i=1}^{\infty} \int_{6^{i} 5 B \backslash 6^{i-1} 5 B}\left|(K(y, z)-K(x, z))\left(b(z)-m_{\sigma^{i_{5} B}}(b)\right)\right| \frac{1}{d(y, z)} d \mu(z) \\
& +c\|f\|_{L^{\infty}(\mu)} \sum_{i=1}^{\infty} \int_{6^{i} 5 B \backslash 6^{i-1} 5 B}\left|(K(y, z)-K(x, z))\left(m_{\sigma^{i_{5} B}}(b)\right)-m_{\widetilde{B}}(b)\right| \frac{1}{d(y, z)} d \mu(z) \\
& =M_{31}+M_{32} \text {. }
\end{aligned}
$$

In Lemma 2.4, we write $a=6^{i} \mu\left(6^{i+1} 5 B\right), t_{1}=\frac{|K(y, z)-K(x, z)|}{d(y, z)}$, and $t_{2}=\frac{\left|b(z)-m_{6^{i_{5}}}\right|}{c_{2}}$. From this we have

$$
\begin{aligned}
& M_{31}(x, y) \leq c\|f\|_{L^{\infty}(\mu)} \sum_{i=1}^{\infty} \int_{6^{i}{ }_{5 B \backslash 6^{i-1} 5 B}} \frac{|K(y, z)-K(x, z)|}{d(y, z)}\left|b(z)-m_{6^{i_{5} B}}(b)\right| d \mu(z) \\
& \leq c\|f\|_{L^{\infty}(\mu)} \sum_{i=1}^{\infty} \int_{6^{i} 5 B \backslash 6^{i-1} 5 B}\left[\frac { | K ( y , z ) - K ( x , z ) | } { d ( y , z ) } \operatorname { l o g } \left[2+6^{i} \mu\left(6^{i+1} 5 B\right)\right.\right. \\
& \left.\left.\times \frac{|K(y, z)-K(x, z)|}{d(y, z)}\right]+\frac{1}{6^{i} \mu\left(6^{i+15 B}\right)} \exp \left(\frac{\left|b(z)-m_{6^{i_{5}}}\right|}{c_{2}}\right)\right] d \mu(z) \\
& \leq c\|f\|_{L^{\infty}(\mu)} \sum_{i=1}^{\infty} i \int_{6^{i} 5 B \backslash 6^{i-1} 5 B} \frac{|K(y, z)-K(x, z)|}{d(y, z)} \log \left(2+\frac{\mu\left(6^{i+1} 5 B\right)}{\lambda\left(c_{B}, d(y, z)\right)}\right) d \mu(z) \\
& +c\|f\|_{L^{\infty}(\mu)} \sum_{i=1}^{\infty} \int_{6^{i} 5 B \backslash 6^{i-1} 5 B} \frac{1}{6^{i} \mu\left(6^{i+1} 5 B\right)} \exp \left(\frac{\left|b(z)-m_{6^{\tilde{i} 5 B}}\right|}{c_{2}}\right) d \mu(z) \\
& \leq c\|f\|_{L^{\infty}(\mu)} \sum_{i=1}^{\infty} i \int_{6^{i} 5 B \backslash 6^{i-1} 5 B} \frac{|K(y, z)-K(x, z)|}{d(y, z)} d \mu(z) \\
& +c\|f\|_{L^{\infty}(\mu)} \sum_{i=1}^{\infty} \frac{1}{6^{i}} \frac{1}{\mu\left(6^{i+1} 5 B\right)} \int_{6^{i} 5 B} \exp \left(\frac{\left|b(z)-m_{6^{\widetilde{i} 5 B}}\right|}{c_{2}}\right) d \mu(z) \\
& \leq c\|f\|_{L^{\infty}(\mu)},
\end{aligned}
$$

where we use (2.3). For $M_{32}$ we estimate

$$
\begin{aligned}
M_{32} & \left.\leq c\|f\|_{L^{\infty}(\mu)} \sum_{i=1}^{\infty} \mid m_{6^{\widetilde{i} 5 B}}(b)\right)-m_{\widetilde{Q}}(b)\left|\int_{6^{i} 5 B \backslash 6^{i-1} 5 B}\right|(K(y, z)-K(x, z)) \mid \frac{1}{d(y, z)} d \mu(z) \\
& \leq c\|f\|_{L^{\infty}(\mu)} \sum_{i=1}^{\infty} i \int_{6^{i} 5 B \backslash 6^{i-1} 5 B}|(K(y, z)-K(x, z))| \frac{1}{d(y, z)} d \mu(z) \\
& \leq c\|f\|_{L^{\infty}(\mu)} .
\end{aligned}
$$


Combining these estimates above, we get

$$
\begin{aligned}
A_{3} & \leq \frac{1}{\mu(6 B)} \int_{B}\left|\mathcal{M}\left(\left(b(y)-m_{\tilde{B}}(b)\right) f_{2}\right)(y)-h_{B}\right| d \mu(y) \\
& \leq c \frac{1}{\mu(6 B)} \frac{1}{\mu(B)} \int_{B} \int_{B} \sum_{i=1}^{3} M_{i}(x, y) d \mu(x) d \mu(y) \\
& \leq c\|f\|_{L^{\infty}(\mu)} .
\end{aligned}
$$

So the estimate (2.4) is proved.

Now we prove (2.5). Consider two balls $B \subset S$ with $x \in B$ and let $N=N_{B, S}+1$, where $S$ is a doubling ball. Write $\left|h_{B}-h_{S}\right|$ as

$$
\begin{aligned}
\mid h_{B}- & h_{S} \mid \\
\leq & \left|m_{S}\left[\mathcal{M}\left(\left(b-m_{\widetilde{B}}(b)\right) f \chi_{\mathcal{X} \backslash 6^{N} B}\right)\right]-m_{B}\left[\mathcal{M}\left(\left(b-m_{\widetilde{B}}(b)\right) f \chi_{\mathcal{X} \backslash 6^{N} B}\right)\right]\right| \\
& +\left|m_{S}\left[\mathcal{M}\left(\left(b-m_{S}(b)\right) f \chi_{\mathcal{X} \backslash 6^{N_{B}}}\right)\right]-m_{S}\left[\mathcal{M}\left(\left(b-m_{\widetilde{B}}(b)\right) f \chi_{\mathcal{X} \backslash 6^{N} B}\right)\right]\right| \\
& +\left|m_{B}\left[\mathcal{M}\left(\left(b-m_{\widetilde{B}}(b)\right) f \chi_{6^{N}{ }_{B \backslash \frac{6}{5}} B}\right)\right]\right|+\left|m_{S}\left[\mathcal{M}\left(\left(b-m_{S}(b)\right) f \chi_{6^{N}}{ } \backslash \frac{6}{5} S\right)\right]\right| \\
= & B_{1}+B_{2}+B_{3}+B_{4} .
\end{aligned}
$$

As in the estimate for the $A_{3}$, we have $B_{1} \leq c\|f\|_{L^{\infty}(\mu)}$. To estimate $B_{2}$, for $y \in \mathcal{X}$, we get

$$
\begin{aligned}
B_{2} & \leq\left|m_{S}\left[\mathcal{M}\left(\left(b-m_{S}(b)\right) f \chi_{\mathcal{X} \backslash 6^{N} B}\right)\right]-m_{S}\left[\mathcal{M}\left(\left(b-m_{\widetilde{B}}(b)\right) f \chi_{\mathcal{X} \backslash 6^{N} B}\right)\right]\right| \\
& \leq c m_{S}\left|\left(m_{S}(b)-m_{\widetilde{B}}(b)\right) \mathcal{M}\left(f \chi_{\mathcal{X} \backslash 6^{N_{B}}}\right)\right| \\
& \leq c \frac{K_{B, S}+K_{B, \widetilde{B}}}{\mu(S)} \int_{S} \mathcal{M}\left(f \chi_{\mathcal{X} \backslash 6^{N_{B}}}\right)(y) d \mu(y) \\
& \leq c \frac{K_{B, S}}{\mu(S)} \mu(S)^{1 / s^{\prime}}\left(\int_{S} \mathcal{M}^{s}\left(f \chi_{\mathcal{X} \backslash 6^{N_{B}}}\right)(y) d \mu(y)\right)^{1 / s} \\
& \leq c K_{B, S} M_{s, 6}[\mathcal{M}(f)] .
\end{aligned}
$$

For $y \in R$, we have

$$
\begin{aligned}
B_{4} & \leq\left|m_{S}\left[\mathcal{M}\left(\left(b-m_{S}(b)\right) f \chi_{6^{N} B \backslash \frac{6}{5} S}\right)\right]\right| \\
& \leq \int_{\mathcal{X}}|K(y, z)|\left|b(z)-m_{S}(b)\right|\left|f(z) \chi_{6^{N} B \backslash \frac{6}{5} S}\right|\left(\int_{d(y, z)<t} \frac{d t}{t^{3}}\right)^{1 / 2} d \mu(z) \\
& \leq c \int_{\mathcal{X}} \frac{\left|b(z)-m_{S}(b)\right|}{\lambda(y, d(y, z))}\left|f(z) \chi_{6^{N} B \backslash \frac{6}{5} S}\right| d \mu(z) \\
& \leq c\|f\|_{L^{\infty}(\mu)} \int_{6^{N} B} \frac{\left|b(z)-m_{S}(b)\right|}{\lambda\left(c_{B}, 6^{N} r_{B}\right)} d \mu \\
& \leq c\|f\|_{L^{\infty}(\mu)} \frac{1}{\lambda\left(c_{B}, 6^{N} r_{B}\right)} \int_{6^{N} B}\left|m_{S}(b)-m_{\widetilde{6 S}}(b)\right|+\left|b(z)-m_{\widetilde{6 S}}\right| d \mu(z) \\
& \leq c\|f\|_{L^{\infty}(\mu)} \frac{1}{\mu\left(6^{2} S\right)} \int_{6 S}\left|b(z)-m_{\widetilde{6} S}\right| d \mu(z)+c\|f\|_{L^{\infty}(\mu)} \mid m_{S}(b)
\end{aligned}
$$




$$
\begin{aligned}
& \quad-m_{\widetilde{6 S}}(b) \mid \frac{\mu\left(6^{N} B\right)}{\lambda\left(c_{B}, 6^{N} r_{B}\right)} \\
& \leq c\|f\|_{L^{\infty}(\mu)},
\end{aligned}
$$

where we have used $\left|m_{S}(b)-m_{\widetilde{6 S}}(b)\right| \leq c\left(K_{S, 6 S}+K_{6 S, \widetilde{6 S}}\right) \leq c$.

In order to estimate $B_{3}$, for $y \in B$, we get

$$
\begin{aligned}
& \left|m_{B}\left[\mathcal{M}\left(\left(b-m_{\widetilde{B}}(b)\right) f \chi_{6^{N} B \backslash \frac{6}{5} B}\right)\right](y)\right| \\
& \leq\left|m_{B}\left[\mathcal{M}\left(\left(b-m_{\widetilde{B}}(b)\right) f \chi_{6^{N} B \backslash 6 B}\right)\right](y)-m_{B}\left[\mathcal{M}\left(\left(b-m_{\widetilde{B}}(b)\right) f \chi_{6 B \backslash \frac{6}{5} B}\right)\right](y)\right| \\
& \leq \int_{6 B \backslash \frac{6}{5} B}|K(y, z)|\left|b(z)-m_{\widetilde{B}}(b)\right||f(z)|\left(\int_{d(y, z)<t} \frac{d t}{t^{3}}\right)^{1 / 2} d \mu(z) \\
& +\int_{6^{N} B \backslash 6 B}|K(y, z)|\left|b(z)-m_{\widetilde{B}}(b)\right||f(z)|\left(\int_{d(y, z)<t} \frac{d t}{t^{3}}\right)^{1 / 2} d \mu(z) \\
& \leq c\|f\|_{L^{\infty}(\mu)} \int_{6 B \backslash \frac{6}{5} B} \frac{\left|b(z)-m_{\widetilde{B}}(b)\right|}{\lambda(y, d(y, z))} d \mu(z)+c \mid f \|_{L^{\infty}(\mu)} \int_{6^{N} B \backslash 6 B} \frac{\left|b(z)-m_{\widetilde{B}}(b)\right|}{\lambda(y, d(y, z))} d \mu(z) \\
& \leq c\|f\|_{L^{\infty}(\mu)} \frac{\mu\left(6^{2} B\right)}{\lambda\left(c_{B}, 6 r_{B}\right)} \frac{1}{\mu\left(6^{2} B\right)} \int_{6 B}\left|b(z)-m_{\widetilde{B}}(b)\right| d \mu(z) \\
& +c\|f\|_{L^{\infty}(\mu)} \sum_{k=1}^{N-1} \int_{6^{k+1} B \backslash 6^{k} B} \frac{\left|b(z)-m_{\widetilde{B}}(b)\right|}{\lambda(y, d(y, z))} d \mu(z) \\
& \leq c\|f\|_{L^{\infty}(\mu)}+c\|f\|_{L^{\infty}(\mu)} \sum_{k=1}^{N-1} \frac{\mu\left(6^{k+2} B\right)}{\lambda\left(c_{B}, 6^{k+1} r_{B}\right)} \frac{1}{\mu\left(6^{k+2} B\right)} \int_{6^{k+1} B}\left|b(z)-m_{6^{k+1} B}(b)\right| d \mu(z) \\
& +c\|f\|_{L^{\infty}(\mu)} \sum_{k=1}^{N-1} \frac{\mu\left(6^{k+2} B\right)}{\lambda\left(c_{B}, 6^{k+1} r_{B}\right)} \frac{1}{\mu\left(6^{k+2} B\right)} \int_{6^{k+1} B}\left|m_{6^{k+1} B}(b)-m_{\widetilde{B}}(b)\right| d \mu(z) \\
& \leq c\|f\|_{L^{\infty}(\mu)}+c\|f\|_{L^{\infty}(\mu)} \sum_{k=1}^{N-1} \frac{\mu\left(6^{k+2} B\right)}{\lambda\left(c_{B}, 6^{k+1} r_{B}\right)}\left(c K_{B, 6^{k+1} B}+1\right) \\
& \leq c K_{B, S}^{2}\|f\|_{L^{\infty}(\mu)} .
\end{aligned}
$$

That is to say, $B_{3} \leq c K_{B, S}^{2}\|f\|_{L^{\infty}(\mu)}$.

Combining the estimates through $B_{1}$ to $B_{4}$ establishes (2.5), which completes the proof of Lemma 2.6.

\section{Proof of Theorem 1.10}

In this section, we prove Theorem 1.10. Let $0<r<1$, we prove that, for any $p \in(1, \infty)$, $b \in L^{\infty}(\mu)$, and all bounded functions $f$ with compact support,

$$
\mu\left(\left\{x \in \mathcal{X}: M_{r}^{\#}\left[\mathcal{M}_{b}(f)\right](x)>\lambda\right\}\right) \leq c \lambda^{-p}\|b\|_{\operatorname{RBMO}(\mu)}^{p}\|f\|_{L^{p}(\mu)}^{p} .
$$

Once (3.1) is established, it follows from the Marcinkiewicz interpolation theorem that

$$
\left\|M_{r}^{\#}\left[\mathcal{M}_{b}(f)\right]\right\|_{L^{p}(\mu)} \leq c\|b\|_{\mathrm{RBMO}(\mu)}\|f\|_{L^{p}(\mu)} .
$$


This via Theorem 4.2 in [25] states that, for any $p \in(1, \infty), b \in L^{\infty}(\mu)$, and all bounded functions $f$ with compact support and integral zero,

$$
\left.\| \mathcal{M}_{b}(f)\right]\left\|_{L^{p}(\mu)} \leq c\right\| b\left\|_{\mathrm{RBMO}(\mu)}\right\| f \|_{L^{p}(\mu)} .
$$

In [25], Theorem 6.4, the authors show the density in $L^{p}(\mu)$ of bounded functions with compact support and integral zero. Similar to [18], Lemma 3.3, using the truncation argument, a routine argument leads to (3.3) for all $b \in \operatorname{RBMO}(\mu)$ and $f \in L^{p}(\mu)$.

Now we prove (3.1). Without loss of generality, we assume that $\rho=6$ in Lemma 2.1 and $\|b\|_{\mathrm{RBMO}(\mu)}=1$. For each fixed $t>0$ and bounded function $f$ with compact support, applying the Calderón-Zygmund decomposition to $|f|^{p}$ at level $t^{p}$ as Lemma 2.2, we decompose $f(x)=g(x)+h(x)$, where

$$
g(x)=f(x) \chi \mathcal{X} \backslash \bigcup_{i} 6 B_{i}(x)+\sum_{i} \varphi_{i}(x), h(x)=\sum_{i}\left[\omega_{i}(x) f(x)-\varphi_{i}(x)\right]=\sum_{i} h_{i}(x) .
$$

It is obvious that $\|g\|_{L^{\infty}(\mu)} \leq c t$. Using Lemma 2.1(2) we have

$$
\begin{aligned}
\left\|\sum_{i} \varphi_{i}\right\|_{L^{p}(\mu)}^{p} & \leq\left\|\sum_{i}\left|\varphi_{i}\right|\right\|_{L^{\infty}(\mu)}^{p-1}\left\|\sum_{i} \varphi_{i}\right\|_{L^{1}(\mu)} \\
& \leq c t^{p-1} \sum_{i}\left(\int_{R_{i}}\left|\varphi_{i}(x)\right|^{p} d \mu(x)\right)^{1 / p} \mu\left(S_{i}\right)^{1 / p^{\prime}} \\
& \leq c \sum_{i} \int_{6 B_{i}}\|f(x)\|^{p} d \mu(x) \leq c\|f\|_{p}^{p} .
\end{aligned}
$$

That is to say, $\|g\|_{L^{p}(\mu)} \leq c\|f\|_{L^{p}(\mu)}$. Using (3.2) and Lemma 2.6 we have

$$
\begin{aligned}
& \mu\left(\left\{x \in \mathcal{X}: M_{r}^{\#}\left(\mathcal{M}_{b}(g)\right)(x)>2 c t\right\}\right) \\
& \quad \leq c \mu\left(\left\{x \in \mathcal{X}: M_{s, 6}(\mathcal{M}(g))(x)>t\right\}\right) \\
& \quad \leq c t^{-p}\left\|M_{s, 6}(\mathcal{M}(g))\right\|_{L^{p}(\mu)}^{p} \leq c t^{-p}\|f\|_{L^{p}(\mu)}^{p},
\end{aligned}
$$

where $1<s<p$.

Similar to [25], Section 4.1, we have, for any $f$,

$$
M_{r}^{\#} f(x) \leq M_{r, 6}(f)(x)+3 N_{r}(f)(x) \leq c M_{r, 6}(f)(x) .
$$

From this we write

$$
\begin{aligned}
\mu\left(\left\{x \in \mathcal{X}: M_{r}^{\#}\left[\mathcal{M}_{b}(h)\right](x)>t\right\}\right) \\
\leq \mu\left(\left\{x \in \mathcal{X}: M_{r, 6}\left[\mathcal{M}\left(\sum_{i}\left(b-m_{\widetilde{6 B_{i}}}(b)\right) h_{i}\right)\right](x)>c t\right\}\right) \\
\quad+\mu\left(\left\{x \in \mathcal{X}: M_{r, 6}\left[\sum_{i}\left|b-m_{\widetilde{6 B_{i}}}(b)\right| \mathcal{M}\left(h_{i}\right)\right](x)>t / 2\right\}\right) \\
=D_{1}+D_{2} .
\end{aligned}
$$


According to the weak type 1-1 estimate for $M_{\rho}$, we have, for any $\lambda>0$,

$$
\lambda \mu\left(\left\{x \in \mathcal{X}: M_{r, 6}(g)(x)>\lambda\right\}\right) \leq c \sup _{\delta>c \lambda} \delta \mu(\{x \in \mathcal{X}:|g(x)|>c \delta\}) .
$$

Taking $1<p_{1}<p$, it follows that

$$
\begin{aligned}
D_{1} & \leq t^{-1} \sup _{\delta>c t} \delta \mu\left(\left\{x \in \mathcal{X}: \mathcal{M}\left(\sum_{i}\left(b-m_{\widetilde{B}}(b)\right) h_{i}\right)(x)>c \delta\right\}\right) \\
& \leq c t^{-p_{1}}\left\|\sum_{i}\left(b-m_{\widetilde{6 B_{i}}}(b)\right) h_{i}\right\|_{L^{p_{1}(\mu)}}^{p_{1}} \\
& \leq c t^{-p_{1}}\left\|\sum_{i}\left(b-m_{\widetilde{6 B_{i}}}(b)\right) f \omega_{i}\right\|_{L^{p_{1}(\mu)}}^{p_{1}}+c t^{-p_{1}}\left\|\sum_{i}\left(b-m_{\widetilde{6 B_{i}}}(b)\right) \varphi_{i}\right\|_{L^{p_{1}(\mu)}}^{p_{1}} \\
& \leq D_{11}+D_{12} .
\end{aligned}
$$

For $D_{11}$, it follows that

$$
\begin{aligned}
D_{11} & \leq c t^{-p_{1}} \sum_{i}\left[\int_{6 B_{i}}|f(x)|^{p} d \mu(x)\right]^{p_{1} / p}\left[\int_{6 B_{i}}\left|b(x)-m_{\widetilde{6 B_{i}}}(b)\right|^{p_{1}\left(p / p_{1}\right)^{\prime}} d \mu(x)\right]^{1-p_{1} / p} \\
& \leq c t^{-p_{1}} \sum_{i}\left[\int_{6 B_{i}}|f(x)|^{p} d \mu(x)\right]^{p_{1} / p} \mu\left(6^{2} B_{i}\right)^{1-p_{1} / p} \\
& \leq c t^{-p_{1}} \sum_{i} \int_{6 B_{i}}|f(x)|^{p} d \mu(x)\left[\int_{6 B_{i}}|f(x)|^{p} d \mu(x)\right]^{p_{1} / p-1} \mu\left(6^{3} B_{i}\right)^{1-p_{1} / p} \\
& \leq c t^{-p}\|f\|_{L^{p}(\mu)}^{p},
\end{aligned}
$$

where we use Lemma 2.2(1). To estimate $D_{12}$, by the fact $\sum_{i} \varphi_{i} \leq c t$, we have

$$
\begin{aligned}
D_{12} \leq & c\left\|\sum_{i}\left(b-m_{\widetilde{6 B_{i}}}(b)\right) \varphi_{i} t^{-1}\right\|_{L^{p_{1}(\mu)}}^{p_{1}} \\
\leq & c\left\|\left[\sum_{i} t^{-1}\left|\varphi_{i}\right|\left|b-m_{\widetilde{6 B_{i}}}(b)\right|^{p_{1}}\right]^{1 / p_{1}}\left[\sum_{i}\left|t^{-1} \varphi_{i}\right|\right]^{1 / p_{1}^{\prime}}\right\|_{L^{p_{1}}(\mu)}^{p_{1}} \\
\leq & c\left\|\left[\sum_{i} t^{-1}\left|\varphi_{i}\right|\left|b-m_{\widetilde{6 B_{i}}}(b)\right|^{p_{1}}\right]^{1 / p_{1}}\right\|_{L^{p_{1}}(\mu)}^{p_{1}} \\
\leq & c t^{-1} \sum_{i} \int_{R_{i}}\left|\varphi_{i}(x)\right|\left|b(x)-m_{\widetilde{6 B_{i}}}(b)\right|^{p_{1}} d \mu(x) \\
\leq & c t^{-1} \sum_{i}\left(\int_{R_{i}}\left|\varphi_{i}(x)\right|^{p} d \mu(x)\right)^{1 / p}\left(\int_{R_{i}}\left|b(x)-m_{\widetilde{R}_{i}}(b)\right|^{p_{1} p^{\prime}} d \mu(x)\right)^{1 / p^{\prime}} \\
& +c t^{-1} \sum_{i} \int_{R_{i}}\left|\varphi_{i}(x)\right|\left|m_{\widetilde{R}_{i}}(b)-m_{\widetilde{6 B_{i}}}(b)\right|^{p_{1}} d \mu(x) \\
\leq & c t^{-1} \sum_{i}\left(\int_{R_{i}}\left|\varphi_{i}(x)\right|^{p} d \mu(x)\right)^{1 / p} \mu\left(R_{i}\right)^{1 / p^{\prime}}+c t^{-1} \sum_{i} \int_{R_{i}}\left|\varphi_{i}(x)\right| d \mu(x)
\end{aligned}
$$




$$
\begin{aligned}
& \leq c t^{-p} \sum_{i} \int_{6 B_{i}}|f(x)|^{p} d \mu(x)+c t^{-1} \sum_{i}\left(\int_{R_{i}}\left|\varphi_{i}(x)\right|^{p} d \mu(x)\right)^{1 / p} \mu\left(S_{i}\right)^{1 / p^{\prime}} \\
& \leq c t^{-p}\|f\|_{L^{p}(\mu)}^{p} .
\end{aligned}
$$

In order to estimate $D_{2}$, we write

$$
\begin{aligned}
D_{2} \leq & c t^{-1} \sum_{i} \int_{\mathcal{X} \backslash 6 S_{i}}\left|b(x)-m_{\widetilde{6 B_{i}}}(b)\right| \mathcal{M}\left(h_{i}\right)(x) d \mu(x) \\
& +c t^{-1} \sum_{i} \int_{6 S_{i}}\left|b(x)-m_{\widetilde{6 B_{i}}}(b)\right| \mathcal{M}\left(\varphi_{i}\right)(x) d \mu(x) \\
& +c t^{-1} \sum_{i} \int_{\frac{6}{5} 6 B_{i}}\left|b(x)-m_{\widetilde{6 B_{i}}}(b)\right| \mathcal{M}\left(\omega_{i} f\right)(x) d \mu(x) \\
& +c t^{-1} \sum_{i} \int_{6 S_{i} \backslash \frac{6}{5} 6 B_{i}}\left|b(x)-m_{\widetilde{6 B_{i}}}(b)\right| \mathcal{M}\left(\omega_{i} f\right)(x) d \mu(x) \\
= & D_{21}+D_{22}+D_{23}+D_{24} .
\end{aligned}
$$

For each $i$, we have

$$
\begin{aligned}
& \int_{\mathcal{X} \backslash 6 S_{i}}\left|b(x)-m_{\widetilde{6 B_{i}}}(b)\right| \mathcal{M}\left(h_{i}\right)(x) d \mu(x) \\
& \quad \leq \int_{\mathcal{X} \backslash 6 S_{i}}\left|b(x)-m_{\widetilde{6 B_{i}}}(b)\right|\left[\int_{0}^{d\left(x, c_{S_{i}}\right)+r_{6 S_{i}}}\left|\int_{d(x, y)<t} K(x, y) h_{i}(y) d \mu(y)\right|^{2} \frac{d t}{t^{3}}\right]^{1 / 2} d \mu(x) \\
& \quad+\int_{\mathcal{X} \backslash 6 S_{i}}\left|b(x)-m_{\widetilde{6 B_{i}}}(b)\right|\left[\int_{d\left(x, c_{i}\right)+r_{6 S_{i}}}^{\infty}\left|\int_{d(x, y)<t} K(x, y) h_{i}(y) d \mu(y)\right|^{2} \frac{d t}{t^{3}}\right]^{1 / 2} d \mu(x) \\
& =D_{21}^{1}+D_{21}^{2} .
\end{aligned}
$$

Using

$$
\left|m_{6^{k+1} 6 S_{i}}(b)-m_{\widetilde{6 B_{i}}}(x)\right| \leq c\left(K_{6 B_{i}, \widetilde{6 B}_{i}}+K_{6 B_{i}, 6 S_{i}}+K_{6 S_{i}, 6^{k+1} 6 S_{i}}+K_{6^{k+1} 6 S_{i},{ }^{k+1} 6 S_{i}}\right) \leq c k,
$$

we get

$$
\begin{aligned}
D_{21}^{1} \leq & \int_{\mathcal{X} \backslash 6 S_{i}}\left|b(x)-m_{\widetilde{6 B_{i}}}(b)\right| \int_{\mathcal{X}}\left|K(x, y) h_{i}(y)\right|\left(\int_{d(x, y)}^{d\left(x, c_{S_{i}}\right)+r_{6 S_{i}}} \frac{d t}{t^{3}}\right)^{1 / 2} d \mu(y) d \mu(x) \\
\leq & c \int_{\mathcal{X} \backslash 6 S_{i}}\left|b(x)-m_{\widetilde{6 B_{i}}}(b)\right| \int_{\mathcal{X}}\left|h_{i}(y)\right| \frac{r_{6 S_{i}}^{1 / 2}}{d\left(x, c_{S_{i}}\right)^{1 / 2} \lambda\left(x, d\left(x, c_{S_{i}}\right)\right)} d \mu(y) d \mu(x) \\
= & c\left\|h_{i}\right\|_{L^{1}(\mu)} \sum_{j} \int_{\sigma^{j+1} 6 S_{i} \backslash \sigma^{j} 6 S_{i}}\left|b(x)-m_{\widetilde{6 B_{i}}}(b)\right| \frac{r_{6 S_{i}}^{1 / 2}}{d\left(x, c_{S_{i}}\right)^{1 / 2} \lambda\left(x, d\left(x, c_{S_{i}}\right)\right)} d \mu(x) \\
\leq & c\left\|h_{i}\right\|_{L^{1}(\mu)} \sum_{j} \int_{\sigma^{j+1} 6 S_{i} \backslash 6_{6} 6 S_{i}}\left|b(x)-m_{\sigma^{j+1} 6 S_{i}}(b)\right| \frac{r_{6 R_{i}}^{1 / 2}}{d\left(x, c_{S_{i}}\right)^{1 / 2} \lambda\left(x, d\left(x, c_{S_{i}}\right)\right)} d \mu(x) \\
& +c\left\|h_{i}\right\|_{L^{1}(\mu)} \sum_{j}\left|m_{\sigma_{\sigma^{j+1} 6 S_{i}}}(b)-m_{\widetilde{6 B_{i}}}(b)\right|
\end{aligned}
$$




$$
\begin{aligned}
& \times \int_{\sigma^{j+1} 6 S_{i} \backslash 6_{6 S_{i}}} \frac{r_{6 S_{i}}^{1 / 2}}{d\left(x, c_{S_{i}}\right)^{1 / 2} \lambda\left(x, d\left(x, c_{S_{i}}\right)\right)} d \mu(x) \\
\leq & c\left\|h_{i}\right\|_{L^{1}(\mu)} \sum_{j}(j+1) \frac{r_{6 S_{i}}^{1 / 2} \mu\left(6^{j+2} 6 S_{i}\right)}{\left(6^{j} r_{6 S_{i}}\right)^{1 / 2} \lambda\left(c_{S_{i}}, 6^{j} r_{6 S_{i}}\right)} \\
\leq & c\left\|h_{i}\right\|_{L^{1}(\mu)} .
\end{aligned}
$$

By the vanishing moment of $h_{i}$, it follows that

$$
\begin{aligned}
D_{21}^{2} \leq & \int_{\mathcal{X} \backslash 6 S_{i}}\left|b(x)-m_{\widetilde{6 B_{i}}}(b)\right| \int_{\mathcal{X}}\left|\left[K(x, y)-K\left(x, c_{R_{i}}\right)\right] h_{i}(y)\right| \\
& \times\left(\int_{d\left(x, c_{S_{i}}\right)+r_{6 S_{i}}}^{\infty} \frac{d t}{t^{3}}\right)^{1 / 2} d \mu(y) d \mu(x) \\
\leq & c \int_{\mathcal{X} \backslash 6 S_{i}}\left|b(x)-m_{\widetilde{6 B_{i}}}(b)\right| \int_{\mathcal{X}}\left|\left[K(x, y)-K\left(x, c_{R_{i}}\right)\right] h_{i}(y)\right| \frac{1}{d\left(x, c_{S_{i}}\right)} d \mu(y) d \mu(x) \\
\leq & \int_{\mathcal{X}} h_{i}(y)\left[\sum_{j} \int_{6^{j+1} 6 S_{i} \backslash 6 \sigma_{6 S_{i}}} \frac{\mid\left[K(x, y)-K\left(x, c_{S_{i}}\right) \mid\right.}{d\left(x, c_{S_{i}}\right)}\right. \\
& \left.\times\left(\left|b(x)-m_{6^{j+1} 6 S_{i}}\right|+\left|m_{6^{j+1} 6 S_{i}}-m_{\widetilde{6 B_{i}}}\right|\right) d \mu(x)\right] d \mu(y) \\
\leq & c\left\|h_{i}\right\|_{L^{1}(\mu)} .
\end{aligned}
$$

But

$$
\begin{aligned}
\left\|h_{i}\right\|_{L^{1}(\mu)} & \leq c\left(\int_{6 B_{i}}|f(x)|^{p} d \mu(x)\right)^{1 / p} \mu\left(6 B_{i}\right)^{1 / p^{\prime}}+\left(\int_{6 S_{i}}|\varphi(x)|^{p} d \mu(x)\right)^{1 / p} \mu\left(6 S_{i}\right)^{1 / p^{\prime}} \\
& \leq c t^{1-p}\|f\|_{L^{p}(\mu)}^{p},
\end{aligned}
$$

so $D_{21} \leq c t^{-1} t^{1-p}\|f\|_{L^{p}(\mu)}^{p} \leq c t^{-p}\|f\|_{L^{p}(\mu)}^{p}$.

For $D_{22}$, it follows from the $L^{p}(\mu)$ boundedness of $\mathcal{M}$ that

$$
\begin{aligned}
D_{22} \leq & c t^{-1} \sum_{i} \int_{6 S_{i}}\left|b(x)-m_{\widetilde{6 B_{i}}}(b)\right| \mathcal{M}\left(\varphi_{i}\right)(x) d \mu(x) \\
& +c t^{-1} \sum_{i}\left|m_{\widetilde{6 B_{i}}}(b)-m_{\widetilde{6 B_{i}}}(b)\right| \int_{6 S_{i}} \mathcal{M}\left(\varphi_{i}\right)(x) d \mu(x) \\
\leq & c t^{-1} \sum_{i}\left(\int_{6 S_{i}}\left|b(x)-m_{\widetilde{6 B_{i}}}(b)\right|^{p^{\prime}} d \mu(x)\right)^{1 / p^{\prime}}\left\|\mathcal{M}\left(\varphi_{i}\right)\right\|_{L^{p}(\mu)} \\
& +c t^{-1} \sum_{i}\left\|\mathcal{M}\left(\varphi_{i}\right)\right\|_{L^{p}(\mu)} \mu\left(6 S_{i}\right)^{1 / p^{\prime}} \\
\leq & c t^{-1} \sum_{i}\left(\int_{S_{i}} \varphi_{i}(x) d \mu(x)\right)^{1 / p} \mu\left(6^{2} S_{i}\right)^{1 / p^{\prime}} \\
\leq & c t^{-1} t^{1-p} \sum_{i} \int_{6 B_{i}}|f(x)|^{p} d \mu(x) \leq c t^{-p}\|f\|_{L^{p}(\mu)}^{p} .
\end{aligned}
$$


Similar to $D_{22}$, we have

$$
\begin{aligned}
D_{23} & \leq c t^{-1} \sum_{i}\left(\int_{\frac{6}{5} 6 B_{i}}\left|b(x)-m_{\widetilde{6 B}_{i}}(b)\right|^{p^{\prime}} d \mu(x)\right)^{1 / p^{\prime}}\left\|\mathcal{M}\left(\omega_{i} f\right)\right\|_{L^{p}(\mu)} \\
& \leq c t^{-1} \sum_{i} \mu\left(S_{i}\right)^{1 / p^{\prime}}\left\|\omega_{i} f\right\|_{L^{p}(\mu)} \\
& \leq c t^{-1} \sum_{i} t^{1-p}\left\|\omega_{i} f\right\|_{L^{p}(\mu)} \int_{6 B_{i}}|f(x)|^{p} d \mu(x)\left(\int_{S_{i}}\left|\varphi_{i}(x)^{p}\right| d \mu(x)\right)^{-1} \\
& \leq c t^{-p}\|f\|_{L^{p}(\mu)} .
\end{aligned}
$$

Next we estimate $D_{24}$. If supp $f \subset B$ for some ball then, for any $\rho>1$ and $x \in \mathcal{X} \backslash \rho B$, we have

$$
\begin{aligned}
\mathcal{M}(f)(x) & \leq c \int_{B}|K(x, y) f(y)|\left(\int_{d(x, y)}^{\infty} \frac{d t}{t^{3}}\right)^{1 / 2} d \mu(y) \\
& \leq c \int_{B} \frac{|f(y)|}{\lambda(x, d(x, y))} \leq \frac{c}{\lambda\left(c_{B}, d\left(x, c_{B}\right)\right)} \int_{B}|f(y)| d \mu(y) .
\end{aligned}
$$

For any $i$ we write $S_{i}=\left(3 \times 6^{2}\right)^{k_{i}} B_{i}$. It follows that

$$
\begin{aligned}
& D_{24} \leq t^{-1} \sum_{i} \int_{6 S_{i} \backslash \frac{6}{5} 6 B_{i}} \frac{\left|b(x)-m_{\widetilde{6 B_{i}}}(b)\right|}{\lambda\left(c_{B_{i}}, d\left(x, c_{B_{i}}\right)\right)} \int_{6 B_{i}}\left|f(y) \omega_{i}(y)\right| d \mu(y) d \mu(x) \\
& \leq c t^{-1} \sum_{i}\left(\int_{6 B_{i}}|f(y)| d \mu(y)\right) \int_{6 S_{i} \backslash S_{i}} \frac{\left|b(x)-m_{\widetilde{6 B_{i}}}(b)\right|}{\lambda\left(c_{B_{i}}, d\left(x, c_{B_{i}}\right)\right)} d \mu(x) \\
& +c t^{-1} \sum_{i}\left(\int_{6 B_{i}}|f(y)| d \mu(y)\right) \int_{S_{i} \backslash \frac{6}{5} 6 B_{i}} \frac{\left|b(x)-m_{\widetilde{6 B_{i}}}(b)\right|}{\lambda\left(c_{B_{i}}, d\left(x, c_{B_{i}}\right)\right)} d \mu(x) \\
& \leq c t^{-1} \sum_{i}\left(\int_{6 B_{i}}|f(y)| d \mu(y)\right) \int_{6 S_{i} \backslash S_{i}} \frac{\left|b(x)-m_{\widetilde{6 S_{i}}}(b)\right|+\left|m_{\widetilde{6 S_{i}}}(b)-m_{\widetilde{6 B_{i}}}(b)\right|}{\lambda\left(c_{B_{i}}, d\left(x, c_{B_{i}}\right)\right)} d \mu(x) \\
& +c t^{-1} \sum_{i}\left(\int_{6 B_{i}}|f(y)| d \mu(y)\right) \\
& \times \sum_{j=0}^{k_{i}-1} \int_{6^{j+1} 6 B_{i} \backslash 6_{6 B_{i}}} \frac{\left|b(x)-m_{\widetilde{6 S_{i}}}(b)\right|+\left|m_{\widetilde{6 S_{i}}}(b)-m_{\widetilde{6 B_{i}}}(b)\right|}{\lambda\left(c_{B_{i}}, d\left(x, c_{B_{i}}\right)\right)} d \mu(x) \\
& \leq c t^{-1} \sum_{i}\left(\int_{6 B_{i}}|f(y)| d \mu(y)\right)\left(1+\frac{\mu\left(6 S_{i}\right)}{\lambda\left(c_{B_{i}}, r_{S_{i}}\right)}\right) \\
& +c t^{-1} \sum_{i}\left(\int_{6 B_{i}}|f(y)| d \mu(y)\right)\left[1+\sum_{j=0}^{k_{i}-1} \frac{\mu\left(6^{j+2} 6 B_{i}\right)}{\lambda\left(c_{B_{i}}, 6^{j+1} r_{B_{i}}\right)} K_{B_{i}, S_{i}}\right] \\
& \leq c t^{-1} \sum_{i}\left(\int_{6 B_{i}}|f(y)| d \mu(y)\right) \leq c t^{-1} \sum_{i}\left(\int_{6 B_{i}}|f(y)|^{p} d \mu(y)\right)^{1 / p} \mu\left(6 B_{i}\right)^{1 / p^{\prime}} \\
& \leq c t^{-p}\|f\|_{L^{p}(\mu)}^{p} \text {. }
\end{aligned}
$$


Combining these estimates for the term $D_{21}, D_{22}, D_{23}$, and $D_{24}$ yields the desired estimate for $D_{2}$. So we complete the proof of Theorem 1.10 .

\section{Competing interests}

The authors declare that they do not have any commercial or associative interest that represents a conflict of interest in connection with the work submitted.

\section{Authors' contributions}

All authors contributed equally to the writing of this paper. All authors read and approved the final manuscript.

\section{Acknowledgements}

Project (Grant No. 11261055, 11161044) supported by NSFC. The authors cordially thank the referees for their careful reading and helpful comments.

Received: 3 March 2015 Accepted: 13 August 2015 Published online: 27 August 2015

\section{References}

1. Marcinkiewicz, J: Sur quelques intégrales du type de Dini. Ann. Soc. Pol. Math. 17, 42-50 (1938)

2. Zygmund, A: Trigonometric Series, 3rd edn. Cambridge University Press, Cambridge (2002)

3. Stein, EM: On the functions of Littlewood-Paley, Lusin, and Marcinkiewicz. Trans. Am. Math. Soc. 88, 430-466 (1958)

4. Ding, Y, Lu, S, Xue, Q: Marcinkiewicz integral on Hardy spaces. Integral Equ. Oper. Theory 42, 174-182 (2002)

5. Ding, Y, Lu, S, Zhang, P: Weighted weak type estimates for commutators of the Marcinkiewicz integrals. Sci. China Ser. A 47, 83-95 (2004)

6. Ding, Y, Xue, Q, Yabuta, K: A remark to the $L^{2}$ boundedness of parametric Marcinkiewicz integral. J. Math. Anal. Appl. 387, 691-697 (2012)

7. $\mathrm{Hu}, \mathrm{G}, \mathrm{Lu}, \mathrm{S}$, Yang, D: $L^{P}\left(R^{m} \times R^{n}\right)$ boundedness for the Marcinkiewicz integral on product spaces. Sci. China Ser. A 46, 75-82 (2003)

8. Hu, G, Meng, Y, Yang, D: Estimates for Marcinkiewicz integrals in BMO and Campanato spaces. Glasg. Math. J. 49, 167-187 (2007)

9. Lu, S: Marcinkiewicz integral with rough kernels. Front. Math. China 3, 1-14 (2008)

10. Torchinsky, A, Wang, S: A note on the Marcinkiewicz integral. Colloq. Math. 60/61, 235-243 (1990)

11. Wu, H: On Marcinkiewicz integral operators with rough kernels. Integral Equ. Oper. Theory 52, $285-298$ (2005)

12. Yang, D, Yang, D, Hu, G: The Hardy Space $H^{1}$ with Non-doubling Measures and Their Applications. Lecture Notes in Mathematics, vol. 2084. Springer, Berlin (2013)

13. Chen, W, Meng, Y, Yang, D: Calderón-Zygmund operators on Hardy spaces without the doubling condition. Proc. Am. Math. Soc. 133, 2671-2680 (2005)

14. Chen, W, Sawyer, E: A note on commutators of fractional integrals with RBMO( $\mu$ ) functions. III. J. Math. 46, 1287-1298 (2002)

15. Tolsa, X: Littlewood-Paley theory and the T(1) theorem with non-doubling measures. Adv. Math. 164, 57-116 (2001)

16. Jiang, Y: Spaces of type BLO for non-doubling measures. Proc. Am. Math. Soc. 133, 2101-2107 (2005)

17. Nazarov, F, Treil, S, Volberg, A: The Tb-theorem on non-homogeneous spaces. Acta Math. 190, 151-239 (2003)

18. Tolsa, X: BMO, $H^{1}$ and Calderón-Zygmund operators for non doubling measures. Math. Ann. 319, 89-149 (2001)

19. Tolsa, X: Painlevé's problem and the semiadditivity of analytic capacity. Acta Math. 190, 105-149 (2003)

20. Yang, D, Zhou, Y: Boundedness of Marcinkiewicz integrals and their commutators in $H^{1}\left(R^{n} \times R^{m}\right)$. Sci. China Ser. A 49 770-790 (2006)

21. Cao, Y, Zhou, J: Morrey spaces for nonhomogeneous metric measure spaces. Abstr. Appl. Anal. 2013, Article ID 196459 (2013). doi:10.1155/2013/196459

22. Coifman, RR, Weiss, G: Extensions of Hardy spaces and their use in analysis. Bull. Am. Math. Soc. 83, 569-645 (1977)

23. Coifman, RR, Weiss, G: Analyse Harmonique Non-commutative sur Certains Espaces Homogènes. Lecture Notes in Mathematics, vol. 242. Springer, Berlin (1971)

24. Hytönen, T: A framework for non-homogeneous analysis on metric spaces and the RBMO space of Tolsa. Publ. Mat. 54, 485-504 (2010)

25. Bui, TA, Duong, XT: Hardy spaces, regularized BMO and the boundedness of Calderón-Zygmund operators on non-homogeneous spaces. J. Geom. Anal. 23, 895-932 (2013)

26. Hytönen, T, Liu, S, Yang, D, Yang, D: Boundedness of Calderón-Zygmund operators on non-homogeneous metric measure spaces. Can. J. Math. 64, 892-923 (2012)

27. Hytönen, T, Martikainen, $\mathrm{H}$ : Non-homogeneous Tb theorem and random dyadic cubes on metric measure spaces. J. Geom. Anal. 22, 1071-1107 (2012)

28. Hytönen, T, Yang, D, Yang, D: The Hardy space $H^{1}$ on non-homogeneous metric spaces. Math. Proc. Camb. Philos. Soc. 153, 9-31 (2012)

29. Liu, S, Yang, D, Yang, D: Boundedness of Calderón-Zygmund operators on non-homogeneous metric measure spaces equivalent characterizations. J. Math. Anal. Appl. 386, 258-272 (2012)

30. Fu, X, Yang, D, Yuan, W: Boundedness on Orlicz spaces for multilinear commutators of Calderón-Zygmund operators on non-homogeneous spaces. Taiwan. J. Math. 16, 2203-2238 (2012)

31. Lin, H, Yang, D: Equivalent boundedness of Marcinkiewicz integrals on non-homogeneous metric measure spaces. Sci. China Math. 57(1), 123-144 (2014)

32. Xing, F, Dachun, Y, Wen, Y: Generalized fractional integrals and their commutators over non-homogeneous metric measure spaces. Taiwan. J. Math. 18(2), 509-557 (2014)

33. Lin, H, Yang, D: Spaces of type BLO on non-homogeneous metric measure spaces. Front. Math. China 6, 271-292 (2011)

34. Hu, G, Lin, H, Yang, D: Marcinkiewicz integrals with non-doubling measures. Integral Equ. Oper. Theory 58, 205-238 (2007) 